\title{
Baby-Friendly Hospital Initiative for Neonatal Wards: impact on breastfeeding practices among preterm infants
}

\author{
Iniciativa Hospital Amigo da Criança para Unidades Neonatais: impacto nas práticas do aleitamento em prematuros
}

Iniciativa Hospital Amigo del Niño para Unidades Neonatales: impacto en las prácticas de lactancia materna en prematuros

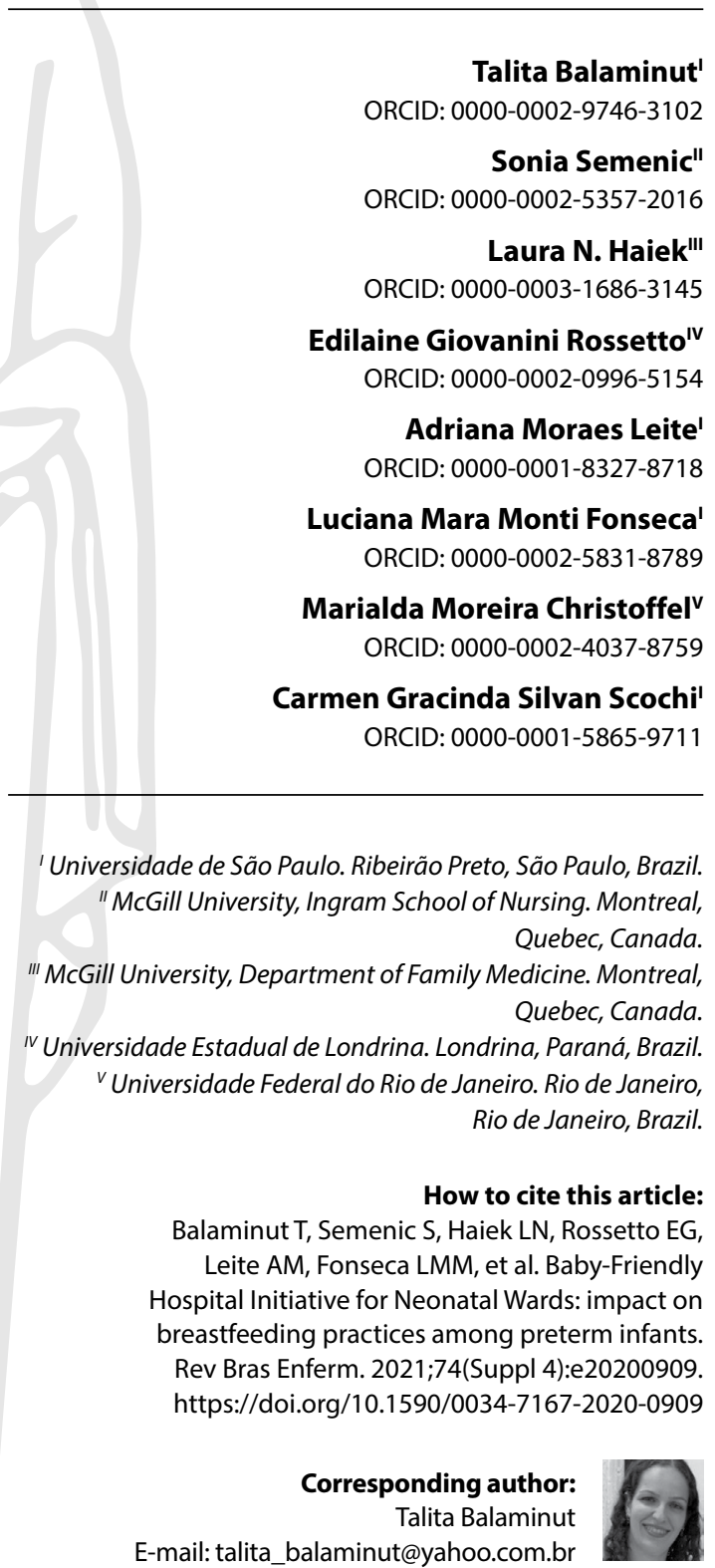

EDITOR IN CHIEF: Dulce Barbosa ASSOCIATE EDITOR: Priscilla Broca

\section{ABSTRACT}

Objective: to assess breastfeeding support practices for preterm infants at two Baby-Friendly hospitals in southeastern Brazil, comparing the effect of implementing the guidelines for Baby-Friendly Hospital Initiative for Neonatal wards. Methods: a quasi-experimental study, pre- and post-intervention with control. Implementation of this initiative in the intervention hospital using Knowledge Translation. Data collection on compliance with the adapted Ten Steps, Three Guiding Principles and the Code before and after the intervention was carried out via interviews with mothers of preterm babies and professionals, unit observation and documentary analysis in the intervention and control hospitals. Intra-intergroup comparison was performed. Results: increases in global compliance with the Three Principles, Ten Steps, the Code, partial compliance with each Principle and in most Steps was greater in the intervention hospital. Conclusion: this initiative improved practices related to breastfeeding in the intervention hospital, demonstrating the potential to improve care and breastfeeding in neonatal wards.

Descriptors: Breast Feeding; Neonatal Intensive Care Units; Infant, Premature; Health Evaluation; Translational Medical Research.

\section{RESUMO}

Objetivo: avaliar as práticas assistenciais do aleitamento materno em prematuros de dois hospitais Amigo da Criança do sudeste brasileiro, comparando o efeito da implementação das diretrizes da Iniciativa Hospital Amigo da Criança para Unidades Neonatais. Métodos: estudo quase-experimental, pré e pós-intervenção com controle. Implementação dessa Iniciativa no hospital intervenção com uso da Knowledge Translation. Coleta da adesão aos Dez Passos adaptados, Três Princípios Norteadores e Código antes e após a intervenção realizadas por entrevistas com mães de prematuros e profissionais, observação das unidades e análise documental nos hospitais intervenção e controle. Realizou-se comparação intra-intergrupos. Resultados: o aumento da adesão global aos Três Princípios e Dez Passos, Código, adesão parcial em cada Princípio e na maioria dos Passos foi maior no hospital intervenção que no controle. Conclusão: essa Iniciativa aprimorou as práticas relacionadas ao aleitamento materno no hospital intervenção, demonstrando potencial em aprimorar a assistência e a amamentação nas Unidades Neonatais.

Descritores: Aleitamento Materno; Unidades de Terapia Intensiva Neonatal; Recém-Nascido Prematuro; Avaliação em Saúde; Pesquisa Médica Translacional.

\section{RESUMEN}

Objetivo: evaluar las prácticas de atención de la lactancia materna en prematuros en dos hospitales Amigo del Niño en el sureste de Brasil, comparando el efecto de la implementación de los lineamientos de la Iniciativa de Hospitales Amigos del Niño para Unidades Neonatales. Métodos: estudio cuasi-experimental, pre y post intervención con grupo control. Implementación de esta iniciativa en el hospital de intervención mediante la traducción del conocimiento. Recolección de adherencia a los Diez Pasos Adaptados, Tres Principios Rectores y Código antes y después de la intervención realizada mediante entrevistas a madres de prematuros y profesionales, observación de las unidades y análisis documentario en los hospitales de intervención y control. Se realizó una comparación intra e intergrupal. Resultados: el aumento en la adherencia global a los Tres Principios, Diez Pasos y el Código asi como la adherencia parcial a cada Principio y en la mayoría de los Pasos fue mayor en el hospital de intervención que en el control. Conclusión: esta iniciativa mejoró las prácticas relacionadas con la lactancia materna en el hospital de intervención, demostrando el potencial para mejorar la atención y la lactancia materna en las Unidades Neonatales. Descriptores: Lactancia Materna; Unidades de Cuidado Intensivo Neonatal; Recién Nacido Prematuro; Evaluación en Salud; Investigación en Medicina Translacional. 


\section{INTRODUCTION}

Establishing and maintaining breastfeeding (BF) in preterm infants in neonatal wards (NW) is complex, presenting greater challenges than in full-term and healthy newborns ${ }^{(1-2)}$. Several factors can make $\mathrm{BF}$ difficult in NWs and have been related to the low rates of $\mathrm{BF}$ in preterm and risk neonates including: fragility, clinical instability and immaturity of preterm infants; maternal behavior in caring for your child; prolonged hospitalization ${ }^{(2)}$; separation between mother and infant; maternal illness and stress; delay in enteral and oral feeding; and insufficient skills of health professionals ${ }^{(3)}$.

There is a gap in international and national public policies related to support for $\mathrm{BF}$ in preterm and critically ill neonates. The Baby-Friendly Hospital Initiative (BFHI) is a hospital intervention strategy to implement practices that promote BF focused on full-term and healthy newborns in maternity wards ${ }^{(4)}$, which also promotes improvements in $\mathrm{BF}$ within $\mathrm{NWs}^{(1,3)}$. However, hospital accreditation by the BFHI does not guarantee the efficiency of support for BF in premature infants admitted to NWs due to its specificities ${ }^{(1,5)}$. Moreover, the BFHI may not be sufficient to elicit professional attitudes and hospital routines favorable to $\mathrm{BF}$ maintenance in this risk group in neonatal units ${ }^{(6)}$.

In an effort to improve BF practices and rates in NWs and, consequently, to reduce morbidity and mortality among preterm and sick neonates, Nordic and Quebec-Canada researchers adapted the BFHI's Ten Steps to Successful Breastfeeding to the needs of $\mathrm{NWs}$, called Neo-BFHII ${ }^{(5,7-8)}$. This expansion includes the adapted Ten Steps, Three Guiding Principles (GP) and, as well as the Code of Marketing of Breast-Milk Substitutes, described in Chart 1.

Implementing the Neo-BFHI's practices may lead to a significant change in the initiation and exclusivity of $\mathrm{BF}$ at discharge and in the empowerment of families to care for their premature children ${ }^{(7)}$. Despite the need for further studies, Neo-BFHI guidelines probably allow more measures to be taken to support $B F$ in $\mathrm{NWs}{ }^{(1)}$ and, consequently, can improve the prevalence of $\mathrm{BF}$ in premature infants ${ }^{(9)}$ and families' experience of their infant's hospitalization, as well as being a strategy to change the culture and philosophy of NWs ${ }^{(10)}$.

Furthermore, Neo-BFHI recommendations are being disseminated in several NWs around the world recently, demonstrating that they are working to support BF. In 2018, 917 NWs in 36 countries participated in a cross-sectional study with the objective of assessing whether they practiced Neo-BFHI recommendations, even without their systematic implementation, through a self-assessment questionnaire, from managers'/health professional's perspectives ${ }^{(11)}$. It was found that these countries partially practiced Neo-BFHI guidelines, with a global compliance of 77 , ranging from 52 to 91 , on a scale of 0 to 100 , with no significant differences between developed and underdeveloped countries and with higher compliance scores in hospitals already accredited in the original $\mathrm{BFH}^{(11)}$.

It is worth noting that few studies have documented policies and practices related to $\mathrm{BF}$ in $\mathrm{NW}^{(1,11)}$. There are no studies that assess the impact of the systematic implementation of Neo-BFHI guidelines on care practices in Brazilian NWs and on the prevalence of $\mathrm{BF}$ among premature infants. Considering the potential of this strategy to promote BF in premature infants admitted to NWs and the absence of research that has assessed the impact of its implementation, this study is linked to a larger national multicenter research, with a focus on assessing compliance with BF support practices in preterm infants through Neo-BFHI implementation.

Chart 1 - Baby-Friendly Hospital Initiative Guidelines for Neonatal wards

\begin{tabular}{|c|c|}
\hline \multicolumn{2}{|c|}{ Guiding principles } \\
\hline $\begin{array}{l}\text { Guiding } \\
\text { principle } 1\end{array}$ & $\begin{array}{l}\text { The staff attitude toward the mother must focus on the } \\
\text { individual mother and her situation. }\end{array}$ \\
\hline $\begin{array}{l}\text { Guiding } \\
\text { principle } 2\end{array}$ & $\begin{array}{l}\text { The facility must provide family-centered care, supported } \\
\text { by the environment. }\end{array}$ \\
\hline $\begin{array}{l}\text { Guiding } \\
\text { principle } 3\end{array}$ & $\begin{array}{l}\text { The health care system must ensure continuity of care, } \\
\text { that is, continuity of pre-, peri-, and postnatal and } \\
\text { postdischarge care. }\end{array}$ \\
\hline \multicolumn{2}{|c|}{$\begin{array}{l}\text { Ten Steps to Successful Breastfeeding Expanded for Neonatal } \\
\text { wards }\end{array}$} \\
\hline Step 1 & $\begin{array}{l}\text { Have a written breastfeeding policy that is routinely } \\
\text { communicated to all health care staff. }\end{array}$ \\
\hline Step 2 & $\begin{array}{l}\text { Educate and train all staff in the specific knowledge and } \\
\text { skills necessary to implement this policy. }\end{array}$ \\
\hline Step 3 & $\begin{array}{l}\text { Inform all hospitalized pregnant women at risk for } \\
\text { preterm delivery or birth of a sick infant about the } \\
\text { management of lactation and breastfeeding and benefits } \\
\text { of breastfeeding. }\end{array}$ \\
\hline Step 4 & $\begin{array}{l}\text { Encourage early, continuous, and prolonged mother- } \\
\text { infant skin-to-skin contact (kangaroo mother care) } \\
\text { without unjustified restrictions. }\end{array}$ \\
\hline Step 5 & $\begin{array}{l}\text { Show mothers how to initiate and maintain lactation and } \\
\text { establish early breastfeeding with infant stability as the } \\
\text { only criterion. }\end{array}$ \\
\hline Step 6 & $\begin{array}{l}\text { Give newborn infants no food or drink other than breast } \\
\text { milk, unless medically indicated. }\end{array}$ \\
\hline Step 7 & $\begin{array}{l}\text { Enable mothers and infants to remain together } 24 \text { hours } \\
\text { a day. }\end{array}$ \\
\hline Step 8 & $\begin{array}{l}\text { Encourage demand feeding or, when needed, semi- } \\
\text { demand feeding as a transitional strategy for preterm and } \\
\text { sick infants. }\end{array}$ \\
\hline Step 9 & $\begin{array}{l}\text { Use alternatives to bottle-feeding at least until } \\
\text { breastfeeding is well established and use pacifiers and } \\
\text { nipple shields only for justifiable reasons. }\end{array}$ \\
\hline Step 10 & $\begin{array}{l}\text { Prepare parents for continued breastfeeding and ensure } \\
\text { access to support services/groups after hospital discharge. }\end{array}$ \\
\hline \multicolumn{2}{|c|}{ International Code of Marketing of Breast Milk Substitutes } \\
\hline
\end{tabular}

Source: Nyqvist KH, Maastrup R, Hansen MN, Häggkvist AP, Hannula L, Ezeonodo A, et al. NeoBFHI:The baby-friendly hospital initiative for neonatal wards. Core document with recommended standards and criteria. Nordic and Quebec Working Group; 2015.

\section{OBJECTIVE}

To assess BF care practices in preterm infants at two BabyFriendly hospitals in southeastern Brazil, comparing the effect of implementing the Baby-Friendly Hospital Initiative guidelines for Neonatal wards.

\section{METHODS}

\section{Ethical aspects}

This research was part of a national multicenter project, which systematically implemented Neo-BFHI guidelines, guided 
by a Knowledge Translation (KT) theoretical framework, in five hospitals - one in each of the Brazilian regions. It was approved by the Research Ethics Committee of the proposing institution. Participants' confidentiality and anonymity were maintained, and they signed an Informed Consent Form.

\section{Design, period, and place of study}

This is a quasi-experimental, controlled intervention study with a pre- and post-test design, guided by SQUIRE. Mixed methods were used to collect data in two hospitals in southeastern Brazil, one intervention hospital $(\mathrm{IH})$ and another control hospital $(\mathrm{CH})$.

Neo-BFHI implementation was carried out only in the IH NW and lasted 16 months (December 2014 to April 2016). Data on compliance with Neo-BFHI guidelines were collected in two moments (moment 1 - M1 and moment 2 - M2) in the two hospitals. In IH, before implementing Neo-BFHl, data collection in $\mathrm{M} 1$ took place from July to September 2014; and in M2, post-intervention, from June to November 2016. In CH, data collection in M1 was carried out from October 2014 to June 2015, and in M2, from August to December 2016.

\section{Tool collection}

Hospital compliance with Neo-BFHI guidelines was assessed by the confidential Neo-BFHI assessment tool, developed by Neo-BFHI international researchers, translated and validated for Brazil and transcribed for data collection on a tablet. In this tool, the scores for compliance with the Neo-BFHI Ten Steps, Three GPs and the Code are a result of the triangulation of data collected in all forms filled out by the interviewer during interviews with mothers of premature infants admitted to NWs (80 questions); health professionals (61 questions) and managers (22 questions) working in these units; and during observations by an external evaluator (NW observation - 21 questions and document analysis - 46 questions).

Steps, GPs and the Code were measured by criteria, with each criterion including the answer to one or more questions in the tool. The tool was prepared to assess compliance with a threshold of $80 \%$ of the responses considered desirable to the criteria. For indicators with less than $80 \%$ of desirable responses, the score received a score of 0 (not implemented); for indicators with $80 \%$ or more of desirable responses, the score received 1 (implemented). Scores were automatically compiled by the electronic data collection form and were composed of partial and global scores for compliance with the Steps and Principles as well as with the Code. Partial scores for compliance with each of the Ten Steps and the Three GPs and the score for compliance with the Code were assessed on a scale of 0 to 1 ; the global score of the Ten Steps (sum of each of the Steps) was assessed on a scale of 0 to 10; the global score of the Three GPs (sum of each $\mathrm{GP}$ ) on a scale of 0 to $3^{(12)}$.

\section{Population or sample; inclusion and exclusion criteria}

A sample calculation was performed to interview mothers of premature infants, also following the Ministry of Health recommendations in the original BFHI assessment. Twenty-one mothers of premature infants hospitalized at least seven days in NWs were interviewed at each moment and in the hospital, with cognitive ability to answer the questionnaires and without temporary or definitive contraindication (maternal or neonatal) to BF. All mothers were chosen via convenience sampling.

The sample of health professionals interviewed in each hospital was composed of $30 \%$ of the total number of professionals in each category. They were drawn among those with direct responsibilities for the care of premature infants, also complying with the Ministry of Health recommendation in the original BFHI assessment. Thus, 41 professionals were interviewed at each moment in $\mathrm{IH}$, and in $\mathrm{CH}, 49$ in $\mathrm{M} 1$ and 47 in $\mathrm{M} 2$. The professionals who answered the questionnaires in $\mathrm{M} 1$ were the same ones who answered in $M 2$, with the exception of those who were no longer in their position at NWs, who were replaced (nine in $\mathrm{IH}$ and 16 in $\mathrm{CH}$ ) by the same professional category and by lot.

The external evaluators in charge of observations and documentary analysis were four trained neonatal nurses. All observations were made in both hospitals on different days and shifts. Documentary analysis included reading and assessing all materials related to BF promotion, protection and support present in NWs (clinical protocols, rules and routines, standard operating procedures, booklets, posters and guidance leaflets, technical reports) in order to complete the tool according to the established criteria.

Moreover, qualitative data related to contextual factors such as human resources staff, training activities carried out, routines and protocols implemented with a focus on BF and renewal of certification in the original BFHI were collected through interviews with heads of NWs and Human Milk Bank (HMB) in both hospitals. In IH, facilitators' impressions (researcher for this study, a nursing professor and a doctoral nurse) were also recorded on the factors that hinder and facilitate the process of implementing the Neo-BFHI Ten Steps.

\section{Study protocol}

Before implementing the intervention in the $\mathrm{IH}(\mathrm{M} 1)$, data were collected on compliance with Neo-BFHI, with data triangulation.

The implementation was guided by a KT theoretical framework $^{(13)}$, guided by the conceptual model PARIHS (Promoting Action on Research Implementation in Health Services) ${ }^{(14)}$, and EPIC strategies (Evidence-Based Practice Identification \& Change) ${ }^{(15)}$. This innovative method from KT voluntarily encourages health professionals to take responsibility for implementing and sustaining changes in clinical practice and assist in routinizing practices that promote an active and responsible participation of mothers/ relatives with the NW multidisciplinary staff.

The process of implementing Neo-BFHI in IH included: training and raising awareness among NW health professionals about Neo-BFHI; voluntary formation of the Research and Practice Council (CPP - Conselho de Pesquisa e Prática), made up of 12 professionals from multidisciplinary staff; organizing data for CPP discussions and consensus on necessary changes according to the Neo-BFHI Ten Steps. The implementation process facilitators were the researcher for this study, a nursing professor, and a doctoral nurse. In rapid cycles, CPP identified the Steps to be worked 
on; the necessary changes; feasible and measurable goals; the indicators for feedback; and the deadlines for goal attainment, through continuous assessment of results and supported by scientific evidence. Four cycles were carried out, lasting three to four months each, in which two or three Steps chosen by CPP were worked on according to priorities.

One month after the end of intervention implementation (M2), data on compliance with Neo-BFHI guidelines were recollected.

There were delays in processing the project at the Research Ethics Committee of the $\mathrm{CH}$, making it impossible to collect data simultaneously on Neo-BFHI compliance at both times in both hospitals. Thus, a minimum interval of 12 months was established between $\mathrm{M} 1$ and $\mathrm{M} 2$ in $\mathrm{CH}$.

Qualitative data related to changes in $\mathrm{CH}$ contextual factors were collected by research assistants every three months, from July 2015 to July 2016, and recorded in a field diary. In IH, such data emerged at CPP meetings, recorded in the field diary by research assistants. In IH, facilitators recorded their impressions of the difficulties and facilitators in the process of implementing the Neo-BFHI Ten Steps to better understand other contextual factors that may have influenced BF practices during the study period.

\section{Analysis of results, and statistics}

Since the hospital which is being investigated (in this study, only two participating hospitals) is considered as the unit of analysis of compliance with Neo-BFHI guidelines, a descriptive intragroup comparison ( $\mathrm{M} 1$ vs $\mathrm{M} 2$ ) in the $\mathrm{IH}$ and $\mathrm{CH}$ as well as intergroup comparison (IH vs $\mathrm{CH}$ ) of changes in compliance scores with the Ten Steps, Three GPs and the Code was performed to assess the effect of the intervention.

For the qualitative data analysis, the field diary notes of both hospitals were summarized and analyzed by a researcher according to the temporality of the events and the hospital compliance data with Neo-BFHI practices. The facilitators'impressions of difficulties and facilities in implementing the Neo-BFHI in IH were also integrated and summarized for interpretation and analysis from the perspective of the multifactorial nature of $B F$ in premature infants, according to each of the Neo-BFHI Ten Steps and GP.

Thus, integrative data analysis was performed by connecting the two methods in the interpretation phase ${ }^{(16)}$. Quantitative data on hospital compliance scores for Neo-BFHI were analyzed more broadly and integrated with the qualitative data.

\section{RESULTS}

In both hospitals, most of the professionals interviewed had a medium level of training ( $\mathrm{M} 1$ and $\mathrm{M} 2=53.7 \%$ in $\mathrm{IH} ; \mathrm{M} 1=46.9 \%$ and $\mathrm{M} 2=27.7 \%$ in $\mathrm{CH}$ ), only one job ( $\mathrm{M} 1=78 \%$ and $\mathrm{M} 2=85.4 \%$ in $\mathrm{IH} ; \mathrm{M} 1=55.1 \%$ and $\mathrm{M} 2=63.8 \%$ in $\mathrm{CH}$ ) and had taken courses or practical training in $\mathrm{BF}$ since joining the neonatal staff $(\mathrm{M} 1=70.7 \%$ and $\mathrm{M} 2=95.1 \%$ in $\mathrm{IH} ; \mathrm{M} 1=87.8 \%$ and $\mathrm{M} 2=93.6 \%$ in $\mathrm{CH})$.

In both hospitals, the mean age of mothers of preterm infants interviewed was similar $(\mathrm{H}=26.7$ and $\mathrm{CH}=28$ years, in $\mathrm{M} 1$ and $\mathrm{M} 2)$, and most of them had eight years or more of study (M1 $=95.2 \%$ and $\mathrm{M} 2=100 \%$ in $\mathrm{IH} ; \mathrm{M} 1=95.2 \%$ and $\mathrm{M} 2=90.5 \%$ in $\mathrm{CH}$ ), a single pregnancy $(\mathrm{M} 1=90.5 \%$ and $\mathrm{M} 2=85.7 \%$ in $\mathrm{IH} ; \mathrm{M} 1=66.7 \%$ and
$\mathrm{M} 2=61.9 \%$ in $\mathrm{CH})$ and cesarean delivery $(\mathrm{M} 1=52.4 \%$ and $\mathrm{M} 2=57$, $1 \%$ in $\mathrm{IH} ; \mathrm{M} 1=81.0 \%$ and $\mathrm{M} 2=76.2 \%$ in $\mathrm{CH}$ ) at 32 weeks or more of gestation $(\mathrm{M} 1=57.1 \%$ and $\mathrm{M} 2=52.4 \%$ in $\mathrm{IH} ; \mathrm{M} 1=52,4 \%$ and $\mathrm{M} 2=76.2 \%$ in $\mathrm{CH}$ ).

Table 1 shows the partial scores for compliance with each of the Three GPs and the expanded Ten Steps to Successful BF, as well as the scores for global compliance with the Three GPs and Ten Steps and compliance with the Code in $\mathrm{IH}$ and $\mathrm{CH}$ in both moments (M1 and M2). After implementing Neo-BFHI, IH demonstrated higher scores for global compliance with the Ten Steps and Three Principles and compliance with the Code than in $\mathrm{CH}$.

Table 1 - Partial and global scores for compliance with the Baby-Friendly Hospital Initiative for Neonatal wards in intervention and control hospitals before (moment 1 ) and after (moment 2 ) the implementation of this initiative in the intervention hospital, Southeast, 2014-2016

\begin{tabular}{|c|c|c|c|c|}
\hline \multirow{2}{*}{$\begin{array}{l}\text { Neo-BFHI compliance scores } \\
\text { (scale from } 0 \text { to } 1 \text { ) }\end{array}$} & \multicolumn{2}{|c|}{$\begin{array}{l}\text { Intervention } \\
\text { Hospital }\end{array}$} & \multicolumn{2}{|c|}{$\begin{array}{c}\text { Control } \\
\text { Hospital }\end{array}$} \\
\hline & M1 & M2 & M1 & M2 \\
\hline GP 1 & 0.50 & 1.00 & 1.00 & 1.00 \\
\hline GP 2 & 0.30 & 0.70 & 0.50 & 0.60 \\
\hline GP 3 & 0.50 & 0.88 & 0.63 & 0.88 \\
\hline Three GP (scale from 1 to 3 ) & 1.30 & 2.58 & 2.13 & 2.48 \\
\hline Step 1 & 0.40 & 0.80 & 0.80 & 0.60 \\
\hline Step 2 & 0.36 & 0.79 & 0.64 & 0.86 \\
\hline Step 3 & 0.80 & 1.00 & 0.60 & 1.00 \\
\hline Step 4 & 0.20 & 0.60 & 0.50 & 0.70 \\
\hline Step 5 & 0.33 & 0.72 & 0.56 & 0.61 \\
\hline Step 6 & 0.33 & 0.22 & 0.33 & 0.56 \\
\hline Step 7 & 0.25 & 0.50 & 0.63 & 1.00 \\
\hline Step 8 & 0.14 & 0.86 & 0.57 & 0.43 \\
\hline Step 9 & 0.15 & 0.62 & 0.31 & 0.23 \\
\hline Step 10 & 0.30 & 0.60 & 0.60 & 0.70 \\
\hline Ten Steps (scale from 0 to 10 ) & 3.27 & 6.70 & 5.54 & 6.68 \\
\hline Code & 0.56 & 0.81 & 0.25 & 0.38 \\
\hline
\end{tabular}

Note: Neo-BFHI - Baby-Friendly Hospital Initiative for Neonatal wards; 11 - moment 1; M2 - moment 2; GP-Guiding Principle.

IH improved compliance scores in all Principles and Steps, except in Step 6, with the difference between scores in $M 1$ and $M 2$, being greater in IH in GP 1, 2 and 3; Three Principles; Steps 1, 2, 4, 5, 8, 9 and 10; Ten Steps; Code. Increases in compliance score were greater in $\mathrm{IH}$ in Steps 3, 6 and 7 when compared with $\mathrm{CH}$. Additionally, in $\mathrm{CH}$, the score decreased in Steps 1,8 and 9 in M2, as shown in Figure 1.

- Intervention Hospital $\quad$ Control Hospital

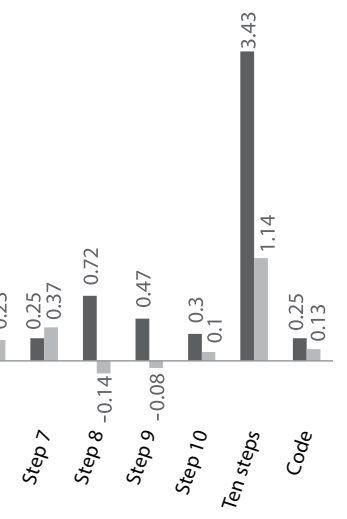

Note: GP - Guiding principle.

Figure 1 - Differences in partial and global scores for compliance with the Baby Friendly Hospital Initiative for Neonatal wards between time 1 and time 2 in intervention and control hospitals. Southeast, 2014-2016 
In $\mathrm{IH}$, there were theoretical-practical training and health education related to BF (Neo-BFHI guidelines, translactation, kangaroo method) as part of the Neo-BFHI implementation process guided by the KT framework. On the other hand, a protocol was implemented in the $\mathrm{IH}$ to control postnatal cytomegalovirus infections, which recommended that raw milk not be given to premature infants born less than 29 weeks for the first eight weeks of life, and premature infants born between 29 and 31 weeks until they complete 35 weeks.

Among the factors recorded by the facilitators influencing the process of implementing $\mathrm{Neo}-\mathrm{BFH}$ in $\mathrm{IH}$, the following stand out: staff discouragement in support actions for BF after the implementation of the above mentioned protocol; difficulty in articulation between sectors and professionals; high workload; little participation by medical staff; professional turnover; difficulties in integrating scientific evidence for changes; difficult acceptance of changes by some professionals.

In $\mathrm{CH}$, we highlight the expansion of $\mathrm{BFH}$ to the global criterion Women Friendly Care after M1. Actions, training and training to promote, protect and support BF were carried out for maternal and child staff; the Ten Step review; and implementation of a continuing education program on BF. In the period between $\mathrm{M} 1$ and $\mathrm{M} 2$, three months after the end of $\mathrm{M} 1$ collection, $\mathrm{CH}$ received a new assessment of the original $\mathrm{BFHI}$ carried out by the Ministry of Health, which did not occur in $\mathrm{IH}$ during the execution of the research, with the previous offer of the BFHI course in the institution.

\section{DISCUSSION}

This study, together with the multicenter project to which it is linked, is the first to partially implement an innovative intervention adapted from the BFHI and extended to the unique needs of NWs in a hospital in southeastern Brazil, and assess hospital compliance with this intervention. This assessment can be considered robust and comprehensive as it includes several perspectives, such as mothers of premature infants, health professionals/managers, document analysis and observation of practices by external evaluators, promoting a holistic view of all practices carried out in NWs. Another study that assessed hospital compliance with Neo-BFHI covered only a single perspective, that of managers/ professionals, through a self-assessment questionnaire ${ }^{(11)}$.

Due to the fact that the two hospitals had different levels of compliance with Neo-BFHI at the beginning of this study (M1) and were not comparable at baseline, it was decided to assess how much compliance with each Step, GP and the Code increased or decreased within each hospital, comparing M1 with M2. From this perspective, it can be determined that Neo-BFHI implementation promoted changes in BF practices in premature infants, helping to improve in-hospital clinical practices in the hospital that received this intervention.

The global compliance score for the Three GPs, on a scale from 0 to 3, stepped from 1.30 to 2.58 in $\mathrm{IH}$, with a greater increase between $M 1$ and $M 2$, and a higher score in $M 2$ in relation to $C H$. Among Principles in IH, GP1 was the one with the highest increase (0.50), followed by GP2 (0.40) and GP3 (0.38). Scores were greater in $\mathrm{IH}$ than in $\mathrm{CH}$. Each of the Three GPs had compliance rates very close to those reported in Brazil in another study, with a score of 88 in GP1, 75 in GP2 and 83 in GP3, on a scale from 0 to $100^{(11)}$.

The increase in global compliance scores with the Ten Steps between $\mathrm{M} 1$ and $\mathrm{M} 2$ was much greater in $\mathrm{IH}$ (3.43) than in $\mathrm{CH}$ (1.14), as well as in global compliance with Three GPs and compliance with the Code, which in $\mathrm{IH}$ there was an increase in the score of 1.28 and 0.25 , while in $\mathrm{CH}$, the increase was 0.35 and 0.13 , respectively. When comparing the difference between each Step before and after implementation in $\mathrm{IH}$, it can be said that there was an increase in all Steps, except in Step 6; while in $\mathrm{CH}$, three Steps (1, 8 and 9) decreased. Furthermore, in IH, the Steps that are most directly related to nursing practices in BF support, such as Steps 4, 5, 8 and 9, showed a considerable increase in compliance scores in M2.

Step 1 had an increase in 0.4 score in $\mathrm{IH}$, while in $\mathrm{CH}$ there was a decrease of 0.20 . Despite the facilitators registering difficulties of professionals in integrating scientific evidence in practices, updates of protocols and written routines of $\mathrm{BF}, \mathrm{IH}$ already presented a written policy on $\mathrm{BF}$ due to accreditation as $\mathrm{BFHI}$, facilitating adaptations to Neo-BFHI and increase of this compliance. Having a written policy for BF in premature infants was one of the organizational factors identified as essential in supporting BF in neonatal intensive care units ${ }^{(17)}$.

Ensuring that health professionals have sufficient, continuous and updated knowledge about BF in NWs is essential (17). BF training during the implementation of $\mathrm{NeO}-\mathrm{BFHI}$ in $\mathrm{IH}$ helped to improve compliance with Step 2, which more than doubled $(0.36$ to 0.79 ) and surpassed the increase in $\mathrm{CH}(0.43$ vs 0.22$)$. However, even with the highest increase in IH scores, $\mathrm{CH}$ obtained a higher compliance score (0.86) with this Step in $\mathrm{M} 2$ when compared with $\mathrm{IH}(0.79)$. This was contextualized by intensification in maternity unit training and qualification related to Women Friendly Care criterion inclusion ${ }^{(4)}$, as well as continuing education program on BF. Compliance with Baby-Friendly practices and commitment to this philosophy of care are directly related to greater professional training ${ }^{(18)}$.

Step 3 has one of the highest scores both hospitals. $\mathrm{CH}$ was the one with the greatest increase $(0.40)$, reaching a maximum compliance score in $\mathrm{M} 2$, which can also be a reflection of continuing education programs and changes in routines and practices related to the expansion of BFHI and Women Friendly Care ${ }^{(4)}$. In $\mathrm{IH}$, an already established routine of daily guidance on lactation/ BF management for hospitalized high-risk pregnant women may account for the high compliance with this Step even before the implementation of Neo-BFHI (0.80), which, when reinforced during implementation, reached a maximum score in M2. Prenatal interventions are effective at increasing rates of BF initiation, duration and exclusivity, especially when combined with interpersonal support and involvement of partner/family ${ }^{(19)}$.

Early, continuous and prolonged skin-to-skin contact (Step 4), an effective and feasible intervention that promotes and supports $\mathrm{BF}^{(3)}$, increased in both hospitals, with $\mathrm{IH}$ showing the highest increase in compliance score; despite tripling numerically (0.20 to 0.60$)$, it was still not enough to exceed $\mathrm{CH}$ in $\mathrm{M} 2(0.70)$. It is noteworthy that only $\mathrm{CH}$ had kangaroo infirmary facilities, allowing continuous skin-to-skin contact, which may justify greater compliance at both times. The considerable increase 
in $\mathrm{IH}$ can be attributed to training and awareness-raising with staff and mothers/relatives about the kangaroo method and the implementation of the electronic record of this practice, facilitating the routine of professionals and boosting its performance.

Step 5 also showed an increase in compliance scores in both hospitals, being more pronounced in $\mathrm{IH}(0.39)$ than in $\mathrm{CH}(0.05)$. The advancement in $\mathrm{IH}$ occurred mainly due to the intense performance of CPP in guiding mothers of premature infants about early and continuous hand expression of milk, and proximity of the HMB collection point to NWs with qualified staff, facilitating practices for maintaining lactation.

A lack of understanding of the mechanisms of lactation, the delay in the initiation of milk expression and the lack of access to information and equipment for expressing milk in NWs are frequent maternal difficulties in the initiation and maintenance of lactation, which can be remedied with guidance from the health staff on the importance, volume, frequency and technique of milk expression ${ }^{(20)}$. Step 5 is especially linked to the responsibility and role of high-risk maternity hospitals that care for women in those early hours, which are essential for the good start and subsequent maintenance of milk production.

Step 6 was the only one in IH that had a decrease of 0.11 , i.e., the compliance score that was already low in M1 (0.33), decreased (0.22), constituting the Step with lowest score after Neo-BFHI implementation. In contrast, in another study, this Step had a much greater compliance (88, on a scale from 0 to 100$)$ in the 51 participating Brazilian units ${ }^{(11)}$, with the exception of this methodological difference between the studies; in the latter, data were informed by institutions' managers.

It is believed that the postnatal cytomegalovirus infection prevention protocol implemented in $\mathrm{IH}$, which recommends not offering raw breast milk to premature infants younger than 30 weeks, can also account for the drop in compliance with Step 6. According to CPP reports, this recommendation led, indirectly, to a decrease in raw breast milk supply for premature infants, and the milk supply that the Human Milk Bank received from mothers of infants admitted to NWs, since the staff was discouraged from supporting early BF and mothers were discouraged from expressing milk early and breastfeeding. In addition to prolonged hospitalization, separation between mother and child, lack of participation in care and fatigue due to the routine of expressing milk at regular and frequent intervals, discouraged mothers of premature infants from milk expression ${ }^{(21)}$. The fact that their raw milk was not offered immediately to their premature infant further discouraged lactation.

Compliance with Step 7 improved in both hospitals, despite the smaller increase in $\mathrm{IH}(0.25)$ compared to $\mathrm{CH}(0.37)$. $\mathrm{CH}$ attained a maximum compliance score and double that obtained by $\mathrm{IH}$ in $\mathrm{M} 2$. In both hospitals, parents have unrestricted access to the units, which was reinforced in $\mathrm{CH}$, where new routines and open access protocols were established, allowing full compliance with $\mathrm{M} 2$.

Despite free access, the full presence of mothers/fathers with their hospitalized children is affected by other factors. In addition to the restrictions imposed by staff, especially at night and during medical visits ${ }^{(17)}$, lack of organization, inadequate physical structure for mothers' needs and changes in family reorganization, especially when having other children, are factors reported by mothers of neonates admitted to $\mathrm{NWs}^{(22)}$. In IH, these aspects also stood out, in addition to the maternal dependence on public transportation with defined schedules, lack of financial conditions to go to the hospital daily and limitations in the distribution of transportation vouchers by the hospital. The complexity of complying with this Step was also demonstrated in another study, with wide variation in compliance between the participating countries (scores from 17 to 100 , on a scale of 0 to 100), and which only $18 \%$ of NW mothers were able to sleep in the same place as their infant during the entire hospitalization ${ }^{(11)}$.

Step 8 obtained the largest increase (0.72) when comparing compliance before and after Neo-BFH in IH. In contrast, in $\mathrm{CH}$, the score for this Step decreased by 0.14 . The improvement in $\mathrm{IH}$ is related to including in the BF policy the issue of using clinical stability as the only criterion to indicate when it is possible to start oral feeding, as well as strategies for semi-demand feeding; greater guidance from mothers on behavioral observation and signs of hunger by infants to position them on the breast; milk supplementation protocol inclusion based on justified medical reasons; and changes in care routines so as not to hinder BF.

As premature infants have particularities and difficulties to promptly follow the recommendation of BF on demand, semi-demand feeding is recommended initially, in response to the behavioral signs of hunger and satiety shown by infants instead of scheduled intervals and with predefined volumes of milk $^{(23)}$. This concept is not yet widely practiced in Brazil, and in $\mathrm{IH}$, despite the changes suggested by CPP, semi-demand food has not been fully implemented. This practice requires mothers' continuous presence in NWs and co-responsibility for feeding their children, with supervision, which are not easily performed in most Brazilian NWs.

Translactation, an effective and more physiological strategy in the transition to oral feeding in preterm infants, was widely discussed in the implementation of Step 9, with theoretical and practical training, which may have contextualized the second largest increase (0.47) among the scores for partial compliance with the Ten Steps in $\mathrm{IH}$. Another strategy that contributed to changes in practices and increased compliance with Step 9 was the formulation of a protocol to use pacifiers for hospitalized premature infants. Due to the fact that it is a Baby-Friendly Hospital, using pacifiers and other nipples was, until then, prohibited $^{(4)}$; however, new evidence has shown the benefits of using a pacifier for premature infants, as long as with well-established indications ${ }^{(6.8,24)}$. When premature infants need support to selfregulate and their mothers are not available/present for suckling at the breast, and when other alternatives for self-regulation are not sufficient, a pacifier can be indicated therapeutically, always under the supervision of a qualified professional and engaged in BF protection ${ }^{(24)}$, including guidance and information for parents on the criteria for its use in the hospital ${ }^{(8)}$.

Finally, the Step 10 compliance score had a greater increase in $\mathrm{IH}(0.30)$ than in $\mathrm{CH}(0.10)$, when comparing the moments, despite the higher $\mathrm{CH}$ score in $\mathrm{M} 2(0.70)$. In the original $\mathrm{BFHI}$, this Step is considered one of the most challenging to implement in hospitals ${ }^{(18)}$, but with the potential to significantly impact BF practices ${ }^{(25)}$, being crucial to maintain support and the best $B F$ rates including the post-discharge period ${ }^{(3,17)}$. Although both hospitals 
have HMB and an outpatient follow-up clinic, more efforts are still needed to ensure continuity of effective comprehensive care for preterm infants and their families.

There was also an increase in compliance with the Code in $\mathrm{IH}$; even though following the recommendations for being Baby Friendly, there is a reinforcement of these guidelines with Neo-BFHI. On the other hand, $\mathrm{CH}$, also accredited, had a very low compliance, not exceeding a score of 0.38 , which even violates the original BFHI. Theoretically, both hospitals should have high compliance with the Code, as they are already accredited as Baby Friendly Hospitals. However, full compliance with the Code has been one of the major challenges in implementing the original $\mathrm{BFHI}$ in various institutions ${ }^{(25)}$. Companies that sell breastmilk substitutes end up exercising influence at various hospital levels, weakening BF protection standards $s^{(3)}$ and making it difficult to implement the BFHI guidelines. This context has been included in the updates to the Ten Steps of the original BFHI, and, in the last revision, the criteria for complying with the Code became part of a substep (Step 1a) in the original $\mathrm{BFHI}^{(25)}$.

Despite the increase in some additions to Neo-BFHI in $\mathrm{CH}$, the global compliance with the Ten Steps and Three GPs and the Code were greater in $\mathrm{IH}$. Neo-BFHI was identified as an intervention that can improve the prevalence of BF in premature infants because it has a holistic approach and in which the relational aspects of care are recognized and integrated, bringing benefits to parents and premature children ${ }^{(9)}$.

It is worth noting the complexity and multifactorial nature of the outcome, and, even if it does not reach full implementation in all Steps and Principles, this innovative intervention, guided by $\mathrm{KT}$, brought about changes and improved clinical practices for the promotion, protection and support of BF in the staff and families of premature infants in IH NWs. The difficulties experienced in the implementation process, as well as the institutional and bureaucratic issues present in $\mathrm{IH}$, can contextualize the incompleteness of implementing the Neo-BFHI, particularly of some Steps. Various scientific evidence to improve lactation and BF during the hospitalization of preterm infants have been detailed in literature, but they are also not widely adopted in clinical practice due to economic barriers (personnel, equipment, materials), ideological ${ }^{(20)}$ and attitudes and behaviors of health professionals ${ }^{(26)}$.

The impact of Neo-BFHI and its sustainability in clinical practice depends on its implementation process and on the commitment of health professionals, i.e., it includes changes in practices and routines, but also in behaviors, attitudes, values, and paradigms. The support provided by the entire health staff was recognized as crucial to implement Neo-BFHI ${ }^{(17)}$. Especially nurses play a significant role in mothers' behavior and decision to breastfeed their premature children; therefore, their attitudes and knowledge in promoting and supporting BF in NWs are essential to successful $\mathrm{BF}^{(26)}$.

\section{Study limitations}

The non-probabilistic sample of mothers of preterm infants interviewed can be considered as a limitation of this study. If, on the one hand, the researcher's participation in data collection and as a facilitator of the intervention implementation process may have created some bias in interpretation and assessment of results, on the other, it may have contributed to better interpretation of the findings given the researcher's knowledge and experience with the reality. Moreover, due to the complexity of implementing the intervention from a horizontal and participatory perspective, this study included only one hospital in which the intervention was performed and a control, which limits generalizations to other hospital contexts.

\section{Contributions to nursing, health, and public policies}

The findings of this study may be relevant for health professionals and mothers who deal with the challenges of BF a premature or critically ill hospitalized infants. Neo-BFHI is an inspiring source for improving care and for professionals to recognize the need to improve their practices. It can also be useful for hospital managers and administrators; to provide advances in national public policies for BF promotion, protection, and support in premature infants; and to encourage the implementation of Neo-BFHI expansion in other Brazilian hospitals in order to guide comprehensive care to this vulnerable population.

\section{CONCLUSION}

The increase in scores of global compliance with the Ten Steps, Three GPs and the Code was greater in the hospital that received the Neo-BFHI intervention when compared before and after its implementation than in $\mathrm{CH}$. Among the partial scores of compliance with Neo-BFHI with greater increase in $\mathrm{IH}$, compared to $\mathrm{CH}$, are Steps 1, 2, 4, 5, 8, 9 and 10, and GP 1, 2 and 3. These steps with greater prominence after the implementation of Neo-BFHI are directly related to the NW's BF policy and the inclusion of mothers in caring for their hospitalized preterm. The only compliance score that decreased in $\mathrm{IH}$ was Step 6 , unlike $\mathrm{CH}$, which had a decrease in the scores of three Steps (1,8 and 9).

Therefore, Neo-BFHI, in this proposal, was relevant to make positive changes in the clinical practice of promoting, protecting and supporting BF in IH NWs. This innovative intervention, implemented in NWs using horizontal and participatory methods of the actors themselves for changes, such as the conceptual model PARIHS and the EPIC strategy, showed a way to improve not only BF, but comprehensive care for premature babies and their families during hospitalization.

\section{FUNDING}

This research was funded by the São Paulo Research Foundation (FAPESP - Fundação de Amparo à Pesquisa do Estado de São Paulo) [2014/10680-1 and 2016/15806-9]. It is part of a multicenter project entitled Aleitamento materno em prematuros: impacto da IHAC para unidades neonatais, funded by the Bill \& Melinda Gates Foundation [OPP1107597], Brazilian National Council for Scientific and Technological Development (Conselho Nacional de Desenvolvimento Científico e Tecnológico) [307401/2014-6 and 401628/2013-2] and Ministry of Health/Department of Science and Technology. 


\section{REFERENCES}

1. Alonso-Díaz C, Utrera-Torres I, Alba-Romero C, Flores-Antón B, Lora-Pablos D, Pallás-Alonso CR. Breastfeeding support in Spanish neonatal intensive care units and the baby-friendly hospital initiative: a national survey. J Hum Lact. 2016;32(4):613-26. https://doi. org/10.1177/0890334416658246

2. Lau C. Breastfeeding challenges and the preterm mother-infant dyad: a conceptual model. Breastfeed Med. 2018;13(1):8-17. https://doi. org/10.1089/bfm.2016.0206

3. Bellù R, Condò M. Breastfeeding promotion: evidence and problems. Pediatr Med Chir. 2017;39(2):53-6. https://doi.org/10.4081/pmc.2017.156

4. Lamounier JA, Chaves RG, Rego MAS, Bouzada MCF. Baby friendly hospital initiative: 25 years of experience in Brazil. Rev Paul Pediatr. 2019;37(4):486-93. https://doi.org/10.1590/1984-0462/;2019;37;4;00004

5. Nyqvist KH, Maastrup R, Hansen MN, Häggkvist AP, Hannula L, Ezeonodo A, et al. Neo-BFHI: The baby-friendly hospital initiative for neonatal wards. Core document with recommended standards and criteria [Internet]. Nordic and Quebec Working Group; 2015 [cited 2020 Apr 15]. Available from: http://epilegothilasmo.gr/wpcontent/uploads/2017/04/Neo_BFHI_Core_document_2015_Edition.pdf

6. Pereira LB, Abrão ACFV, Ohara CVS, Ribeiro CA. Maternal experiences with specificities of prematurity that hinder breastfeeding. Texto Contexto Enferm. 2015;24(1):55-63. https://doi.org/10.1590/0104-07072015000540014

7. Nyqvist KH, Häggkvist AP, Hansen MN, Kylberg E, Frandsen AL, Maastrup R, et al. Expansion of the ten steps to successful breastfeeding into neonatal intensive care: expert group recommendations for three guiding principles. J Hum Lact. 2012;28(3):289-96. https://doi. org/10.1177/0890334412441862

8. Nyqvist KH, Häggkvist AP, Hansen MN, Kylberg E, Frandsen AL, Maastrup R, et al. Expansion of the baby-friendly hospital initiative ten steps to successful breastfeeding into neonatal intensive care: expert group recommendations. J Hum Lact. 2013;29(3):300-9. https://doi. org/10.1177/0890334413489775

9. Ericson J, Flacking R, Hellström-Westas L, Eriksson M. Changes in the prevalence of breastfeeding in preterm infants discharged from neonatal units: a register study over 10 years. BMJ Open. 2016;6(12):e012900. https://doi.org/10.1136/bmjopen-2016-012900

10. Read K, Rattenbury L. Parents as partners in care: lessons from the Baby Friendly Initiative in Exeter. J Neonatal Nurs. 2018;24(1):17-20. https://doi.org/10.1016/j.jnn.2017.11.006

11. Maastrup R, Haiek LN, Neo-BFHI Survey Group. Compliance with the "Baby-friendly hospital initiative for neonatal wards" in 36 countries. Matern Child Nutr. 2019;15(2):e12690. https://doi.org/10.1111/mcn.12690

12. Haiek LN. Measuring compliance with the Baby-Friendly Hospital Initiative. Public Health Nutr. 2012;15(5):894-905. https://doi.org/10.1017/ S1368980011002394

13. Canadian Institutes of Health Research (CIHR). More about knowledge translation at CIHR: knowledge translation definition [Internet]. Ottawa (ON): Government of Canada; 2016 [cited 2020 Sep 21]. Available from: https://cihr-irsc.gc.ca/e/29418.html\#7

14. Bandeira AG, Witt RR, Lapão LV, Madruga JG. A utilização de um referencial metodológico na implementação de evidências como parte da investigação em enfermagem. Texto Contexto Enferm. 2017;26(4):e25550017. https://doi.org/10.1590/0104-07072017002550017

15. Lee SK, Aziz K, Singhal N, Cronin CM, James A, Lee DSC, et al. Improving the quality of care for infants: a cluster randomized controlled trial. CMAJ. 2009;81(8):469-76. https://doi.org/10.1503/cmaj.081727

16. Creswell JW, Creswell JD. Research design: qualitative, quantitative, and mixed methods approaches. 5nd ed. Thousand Oaks: Sage Publications; 2017. 304 p.

17. Tambani E, Giannì ML, Bezze EN, Sannino P, Sorrentino G, Plevani L, et al. Exploring the gap between needs and practice in facilitating breastfeeding within the neonatal intensive care setting: an Italian survey on organizational factors. Front Pediatr. 2019;7:276. https://doi. org/10.3389/fped.2019.00276

18. Munn AC, Newman SD, Mueller M, Phillips SM, Taylor SN. The impact in the United States of the baby-friendly hospital initiative on early infant health and breastfeeding outcomes. Breastfeed Med. 2016;11(5):222-30. https://doi.org/10.1089/bfm.2015.0135

19. Wouk K, Tully KP, Labbok MH. Systematic review of evidence for baby-friendly hospital initiative step 3. J Hum Lact. 2017;33(1):50-82. https://doi.org/10.1177/0890334416679618

20. Meier PP, Johnson TJ, Patel AL, Rossman B. Evidence-based methods that promote human milk feeding of preterm infants: an expert review. Clin Perinatol. 2017;44(1):1-22. https://doi.org/10.1016/j.clp.2016.11.005

21. Medina IMF, Fernández-Sola C, López-Rodríguez MM, Hernández-Padilla JM, Lasserrotte MMJ, Granero-Molina J. Barriers to providing mother's own milk to extremely preterm infants in the NICU. Adv Neonatal Care. 2019;19(5):349-60. https://doi.org/10.1097/ ANC.0000000000000652

22. Zanfolim LC, Cerchiari EAN, Ganassin FMH. Difficulties experienced by mothers during the hospitalization of their babies at neonatal units. Psicol Cienc Prof. 2018;38(1):22-35. https://doi.org/10.1590/1982-3703000292017

23. Lubbe W. Clinicians guide for cue-based transition to oral feeding in preterm infants: an easy-to-use clinical guide. J Eval Clin Pract. 2018;24(1):80-8. https://doi.org/10.1111/jep.12721 
24. Lubbe W, Ham-Baloyi WT. When is the use of pacifiers justifiable in the baby-friendly hospital initiative context? a clinician's guide. BMC Pregnancy and Childbirth. 2017;17(1):130. https://doi.org/10.1186/s12884-017-1306-8

25. World Health Organization (WHO). Implementation guidance - protecting, promoting and supporting breastfeeding in facilities providing maternity and newborn services: the revised Baby-Friendly Hospital Initiative [Internet]. Geneva: World Health Organization, Department of Nutrition for Health and Development; 2018 [cited 2020 May 05]. Available from: https://www.who.int/nutrition/publications/ infantfeeding/bfhi-implementation-2018.pdf

26. Shattnawi KK. Healthcare professionals' attitudes and practices in supporting and promoting the breastfeeding of preterm infants in NICUs. Adv Neonatal Care. 2017;17(5):390-9. https://doi.org/10.1097/ANC.0000000000000421 